\title{
Gravity as a constrained BF theory: Noether charges and Immirzi parameter
}

\author{
R. Durka* and J. Kowalski-Glikman ${ }^{\dagger}$ \\ Institute for Theoretical Physics, University of Wroctaw, Pl. Maxa Borna 9, Pl-50-204 Wroctaw, Poland
}

(Dated: September 19, 2018)

\begin{abstract}
We derive and analyze Noether charges associated with the diffeomorphism invariance for the constrained $S O(2,3) \mathrm{BF}$ theory. This result generalizes the Wald approach to the case of the first order gravity with a negative cosmological constant, the Holst modification and topological terms (Nieh-Yan, Euler, and Pontryagin). We show that differentiability of the action is automatically implemented by the the structure of the constrained BF model. Finally, we calculate the AdSSchwarzschild black hole entropy from the Noether charge and we find that it does not depend on the Immirzi parameter.
\end{abstract}

\section{INTRODUCTION}

The Wilsonian perspective is a powerful guiding principle in constructing theories with the given field content and symmetries. It tells that one should include in the action all terms that can be constructed from the fields and are compatible with the symmetries of the theory. In the context of first order gravity we have to do with two fields, tetrad $e^{a}$ and connection $\omega^{a b}$, and two symmetries, local Lorentz invariance and spacetime diffeomorphisms. If we implement the diffeomorphism invariance, assuming that the action of gravity is written as a four form polynomial constructed from the tetrad and the connection, the list of possible terms turns out to be rather short and includes

- Palatini Lagrangian

$$
\mathcal{L}_{P}=R^{a b} \wedge e^{c} \wedge e^{d} \epsilon_{a b c d}
$$

- Cosmological term

$$
\mathcal{L}_{C}=e^{a} \wedge e^{b} \wedge e^{c} \wedge e^{d} \epsilon_{a b c d},
$$

- Holst term 12

$$
H_{4}=R^{a b} \wedge e_{a} \wedge e_{b},
$$

- Pontryagin, Euler and Nieh-Yan topological terms

$$
\begin{aligned}
P_{4} & =R^{a b} \wedge R_{a b}, \\
E_{4} & =R^{a b} \wedge R^{c d} \epsilon_{a b c d}, \\
N Y_{4} & =T^{a} \wedge T_{a}-R^{a b} \wedge e_{a} \wedge e_{b},
\end{aligned}
$$

where $R^{a b}$ is the curvature of $\omega^{a b}$ and $T^{a}$ is torsion.

Each of these terms comes with its own coupling constant. One could ask if there is an additional principle that could be used to reduce the number of independent parameters of the theory. As it turns out, this can be achieved in the framework of the formulation of gravity as a constrained BF theory.

This approach has its roots in MacDowell-Mansouri [1], 2] and Plebanski [3 5] theories and was developed in the series of papers [6 9]. In this formulation we have the anti-de Sitter

\footnotetext{
* rdurka@ift.uni.wroc.pl

† jkowalskiglikman@ift.uni.wroc.pl
}

algebra $s o(2,3)$-valued 1 connection $A^{I J}$, with $I, J=0, \ldots, 4$, which can be decomposed into Lorentz connection $\omega^{a b}$ and the tetrad (soldering) one-form $e^{a}(a, b=0, \ldots, 3)$ as follows

$$
A^{a b}=\omega^{a b}, \quad A^{a 4}=\frac{1}{\ell} e^{a} .
$$

Here $\ell$ is a length scale necessary for dimensional reason since the tetrad is dimensionless. As we will see this scale is naturally associated with the cosmological constant. The components of the curvature of connection $A^{I J}$ are related to the curvature of Lorentz connection $\omega$

$$
F^{a b}(A)=R^{a b}(\omega)+\frac{1}{\ell^{2}} e^{a} \wedge e^{b}
$$

and the torsion

$$
F^{a 4}=\frac{1}{\ell}\left(d e^{a}+\omega_{b}^{a} \wedge e^{b}\right)=\frac{1}{\ell} T^{a} .
$$

With the help of the second ingredient, the $s o(2,3)$ Lie algebra valued two-from field $B^{I J}$ one can write down the action of the theory as follows

$16 \pi S(A, B)=\int F^{I J} \wedge B_{I J}-\frac{\beta}{2} B^{I J} \wedge B_{I J}-\frac{\alpha}{4} \epsilon^{I J K L 4} B_{I J} \wedge B_{K L}$

After solving $B$ field equations we find

$$
B^{a 4}=\frac{1}{\beta} F^{a 4}, \quad B^{a b}=\frac{1}{2\left(\alpha^{2}+\beta^{2}\right)}\left(\beta \delta_{c d}^{a b}-\alpha \epsilon_{c d}^{a b}\right) F^{c d} .
$$

Before substituting this result back to the action (1.8) let us provide the expressions for dimensionless coupling constants $\alpha$ and $\beta$ and the scale $\ell$ in terms of the physical coupling constants, Newton's constant $G$, a negative cosmological constant $\Lambda$, and the Immirzi parameter $\gamma[10]$

$\alpha=\frac{G \Lambda}{3} \frac{1}{\left(1+\gamma^{2}\right)}, \quad \beta=\frac{G \Lambda}{3} \frac{\gamma}{\left(1+\gamma^{2}\right)}, \quad \gamma=\frac{\beta}{\alpha}, \quad \Lambda=-\frac{3}{\ell^{2}}$.

\footnotetext{
1 The de Sitter case so $(1,4)$ can be constructed analogously. Here we use the anti-de Sitter algebra because it leads to the asymptotically anti-de Sitter spacetimes.
} 
Substituting (1.9) and (1.10) to the action (1.8) gives

$$
\begin{aligned}
32 \pi G S= & \int\left(R^{a b} \wedge e^{c} \wedge e^{d}+\frac{1}{2 \ell^{2}} e^{a} \wedge e^{b} \wedge e^{c} \wedge e^{d}\right) \epsilon_{a b c d} \\
& +\frac{2}{\gamma} \int R^{a b} \wedge e_{a} \wedge e_{b} \\
& +\frac{\ell^{2}}{2} E_{4}-\ell^{2} \gamma P_{4}+\frac{2\left(\gamma^{2}+1\right)}{\gamma} N Y_{4}
\end{aligned}
$$

The first line in (1.11) is the standard first order form of the general relativity action with the cosmological constant. The third line contains the combination of topological invariants and therefore can be written as a total derivative (see [1]]). The middle term is called the Holst term 12]. Although it is not a total derivative, by the virtue of the Bianchi identity, it does not influence equations of motion when torsion vanishes.

The first order action above can be also written down in a compact form

$$
S(\omega, e)=\frac{1}{16 \pi} \int_{M}\left(\frac{1}{4} M^{a b c d} F_{a b} \wedge F_{c d}-\frac{1}{\beta \ell^{2}} T^{a} \wedge T_{a}\right)
$$

with

$$
M^{a b}{ }_{c d}=\frac{\alpha}{\left(\alpha^{2}+\beta^{2}\right)}\left(\gamma \delta_{c d}^{a b}-\epsilon_{c d}^{a b}\right) \equiv-\frac{\ell^{2}}{G}\left(\gamma \delta_{c d}^{a b}-\epsilon_{c d}^{a b}\right) .
$$

The field equations following from (1.12) read

$$
\begin{aligned}
K^{a b c d} F_{a b} \wedge e_{c} & =0 \\
\frac{1}{\ell^{2}} \mathcal{D}^{\omega}\left(K^{a b c d}\left(e_{a} \wedge e_{b}\right)\right) & =0
\end{aligned}
$$

where the operator $K$ has the form

$$
K^{a b}{ }_{c d} \equiv-\frac{\ell^{2}}{G}\left(\frac{1}{\gamma} \delta_{c d}^{a b}+\epsilon^{a b}{ }_{c d}\right)
$$

and we have introduced AdS curvature $F$

$$
F^{a b}=\left(R^{a b}+\frac{1}{\ell^{2}} e^{a} \wedge e^{b}\right)
$$

Later we will make use of the fact that this curvature vanishes for anti-de Sitter spacetime.

It follows from equation (1.15) that torsion $T^{a}=\mathcal{D}^{\omega} e^{a}$ vanishes (one has to assume that $\gamma^{2} \neq-1$ ) and thus the field equations (1.16) are Einstein equations with a negative cosmological constant in the first order form.

The Immirzi parameter, being the coupling constant associated with the Holst term, is a mysterious beast. It was first introduced by Barbero [13] in the context of Ashtekar variables, parametrizing a family of canonical transformations on the gravity phase space and inequivalent quantizations. It was soon realized that $\gamma$ is explicitly present in the Loop Quantum Gravity formula for area spectrum [10], [14]. As a consequence, Immirzi parameter is also present in the formula for black hole entropy calculated by counting LQG microstates of isolated horizon [15 19]; for the recent discussion of the results see [20]. On the other hand, as it was said above, the inclusion of the Holst term does not lead to any modifications of classical field equations of gravity and therefore is seemingly completely irrelevant classically.

However, it is well known that black hole entropy can be computed [21], 22] in a class of diffeomorphism invariant theories as a Noether charge associated with a timelike Killing vector with a vanishing norm at the horizon.
A natural question arises: if we calculate the black hole entropy following the Wald and Iyer recipe in the theory of gravity with Holst term, will we reproduce the Loop Quantum Gravity result? This is the main problem we would like to address in this paper.

The BF formulation of gravity is a very convenient starting point in this context. First, it naturally leads to the emergence of the Holst term. Second, the analysis of the boundary terms is particularly simple in this case. As we will see below, in this formulation the problem of notorious counterterms, that usually have to be added to the action in order to make it differentiable and finite, is automatically taken care of. Last, but not least, the calculation of Noether charges in this formulation is much simpler than in the case of the standard first order gravity.

The plan of this paper is as follows. In the next section we will show that in a black hole, asymptotically anti-de Sitter spacetime, the action (1.8) is differentiable. This remarkable fact can be understood in the complementary first order gravity formulation as being due to the presence of the topological invariants with the right coefficients. In Sec. III, returning to the constrained BF theory, we will construct the Noether charges following the construction of Wald and Iyer [21], 22]. Next, in Sec. IV we make use of these expressions to calculate entropy of Schwarzschild-AdS black hole. The final section will be devoted to discussion and conclusions.

\section{BOUNDARIES AND DIFFERENTIABILITY}

When spacetime has boundaries we must make sure that the action (1.8) is differentiable and the variational principle is well defined 2. The differentiability of the action means essentially that the values of the fields and the form of variations are chosen in such a way that the boundary contribution to the variation of the action vanishes. Investigating this we will see how powerful is the BF formulation outlined in the previous section. In what follows we will restrict ourselves to the black hole spacetimes with the anti-de Sitter asymptotic; therefore we will have to do with a manifold with the boundary at infinity, where the gravitational field satisfies $F^{a b}=0$ (cf.1.17), and the inner black hole boundary, where we assume that the variation of connection vanishes $\delta \omega^{a b}=0$. This latter condition is imposed because fixing connection at the horizon means fixing the black hole temperature, and therefore this boundary condition is essentially equivalent to imposing the zeroth law of black hole mechanics.

Consider the variation of the action (1.8) keeping only the terms that contribute to the boundary integral

$$
\begin{aligned}
16 \pi \delta S(A, B) & =\int_{M} \delta F^{I J} \wedge B_{I J}+\ldots=\int_{M} d \delta A^{I J} \wedge B_{I J}+\ldots \\
& =\int_{\partial M} \delta A^{I J} \wedge B_{I J}+\text { bulk terms }
\end{aligned}
$$

with $\partial M=\left(\mathbb{R} \times \partial \Sigma_{\infty}\right) \cup\left(\mathbb{R} \times \partial \Sigma_{H}\right)$.

\footnotetext{
2 Usually one also assumes that the action should be finite for physically reasonable asymptotic conditions for the fields at infinity, so as to make the path integral meaningful. We will not investigate this issue in details here.
} 
There are two contributions to the integral at infinity, proportional to $B_{a 4}$ and $B_{a b}$. The first vanishes because $B_{a 4}$ is proportional to torsion which vanishes by the field equations, and the second is zero because $B_{a b} \sim F_{a b}$ which vanishes by the virtue of asymptotic condition.

Similarly, at the black hole horizon the term $\delta A^{a 4} \wedge B_{a 4}$ is proportional to torsion and therefore zero, while the term $\delta A^{a b} \wedge B_{a b}$ vanishes because we choose the boundary condition $\delta \omega^{a b}=0$ there, as discussed above. Therefore, remarkably, we find that the BF action is differentiable without any need of adding counterterms.

To understand how this result comes about let us notice that the action (1.8) written in the components has the form 3

$$
\left[(\text { Palatini }+\Lambda)+\ell^{2} \text { Euler }\right]-\gamma\left[\text { Holst }+\ell^{2} \text { Pontryagin }\right]
$$

It can be checked that these are exactly the combinations needed to cancel out the boundary terms at infinity resulting from varying the Palatini and Holst actions. To see this consider the first combination above. Take an arbitrary variation of the Palatini action, to wit

$$
\delta(\text { Palatini }+\Lambda)=\int_{M}(\text { f.e. })_{a} \delta e^{a}+(\text { f.e. })_{a b} \delta \omega^{a b}+\int_{\partial M} \Theta
$$

where (f.e.) denote field (Einstein and torsion) equations, while

$$
\Theta=\frac{1}{32 \pi G} \epsilon_{a b c d} \delta \omega^{a b} \wedge e^{c} \wedge e^{d} .
$$

Let us now turn to the Euler term. As it is well known

$$
E_{4}=32 \pi \chi(M)+2 \int_{\partial M} \widetilde{C S}_{3},
$$

where $\chi(M)$ is the Euler characteristics of the manifold $M$ and $\widetilde{C S}_{3}$ is the Chern-Simons three-form for the Lorentz gauge algebra. The Euler characteristics is a fixed number and its variation vanishes; the variation of Chern-Simons form is

$$
\delta \widetilde{C S}_{3}=\epsilon_{a b c d} \delta \omega^{a b} \wedge R^{c d} .
$$

It can be now checked directly that the terms (2.3) and 2.5) are being combined to give $\delta \omega^{a b} \wedge F^{c d} \epsilon_{a b c d}$, which is zero by the virtue of the asymptotic condition at infinity, and by boundary condition at the horizon. The Holst term and the Pontryagin counterterm can be analyzed similarly.

\section{NOETHER CHARGES AND ENTROPY}

Now knowing that the action (1.8) is differentiable we can turn to the discussion of the Noether charges associated with its symmetries. In our derivation below we will follow the procedure proposed in the papers [21] and [22]. Let us start with an arbitrary variation of the action (1.8)

$$
\begin{aligned}
& 16 \pi \delta S=\int( \delta B^{I J} \wedge\left(F_{I J}-\beta B_{I J}-\frac{\alpha}{2} B^{K L} \epsilon_{I J K L 4}\right)+ \\
&\left.+\delta A_{I J} \wedge\left(\mathcal{D}^{A} B^{I J}\right)+d\left(B^{I J} \wedge \delta A_{I J}\right)\right)
\end{aligned}
$$

3 The prefactor $\gamma$ results from combining the original Holst term with torsionless part of Nieh-Yan term.
The expressions proportional to the variations of $B^{I J}$ and $A^{I J}$ in the bulk are field equations, while the last term is the total derivative of the 3 -form symplectic potential:

$$
\Theta=B^{I J} \wedge \delta A_{I J}
$$

For an arbitrary diffeomorphism generated by a smooth vector field $\xi^{\mu}$, one can derive the conserved Noether current 3-form $J$ given by

$$
J[\xi]=\Theta\left[\phi, L_{\xi} \phi\right]-I_{\xi} \mathcal{L}, \quad J[\xi]=B^{I J} \wedge L_{\xi} A_{I J}-I_{\xi} \mathcal{L}
$$

where $\mathcal{L}$ is the Lagrangian, $L_{\xi}$ denotes the Lie derivative in the direction $\xi$ and contraction $I_{\xi}$ (acting on a $p$-form $\alpha$ ) is defined to be

$$
I_{\xi} \alpha_{p}=\frac{1}{(p-1) !} \xi^{\mu} \alpha_{\mu \nu^{1} \ldots \nu^{p-1}} d x^{\nu^{1}} \wedge \cdots \wedge d x^{\nu^{p-1}}
$$

By direct calculation we find

$$
\begin{aligned}
16 \pi J[\xi] & =\left(F_{I J}-\beta B_{I J}-\frac{\alpha}{2} B^{K L} \epsilon_{I J K L 4}\right) \wedge I_{\xi} B_{I J} \\
& +I_{\xi} A_{I J} \wedge\left(\mathcal{D}^{A} B^{I J}\right)+d\left(B^{I J} \wedge I_{\xi} A_{I J}\right) .
\end{aligned}
$$

When field equations are satisfied this current is an exact differential of a two form and thus we can write down the associated charge to be

$$
Q[\xi]=\frac{1}{16 \pi} \int_{\partial \Sigma} B^{I J} I_{\xi} A_{I J}
$$

which, after substituting the solution of the $B$ field equations takes the form

$$
Q=\frac{1}{16 \pi} \int_{\partial \Sigma}\left(\frac{1}{2} M^{a b}{ }_{c d} F_{a b} I_{\xi} \omega^{c d}-\frac{2}{\beta \ell^{2}} T_{a} I_{\xi} e^{a}\right),
$$

where $\partial \Sigma$ is a spatial section of the manifold.

One can check that the expression for the Noether charge (3.4) agrees with the one that can be obtained from the first order action (1.12), as it should. It is also worth noticing that the Noether charge can be expressed compactly as

$$
Q=\frac{1}{16 \pi} \int_{\partial \Sigma} \frac{\delta \mathcal{L}}{\delta F^{I J}} I_{\xi} A^{I J}
$$

Turning back to the formula (3.4) and taking torsion $T^{a}=0$ we can express the charge in the final form

$$
Q[\xi]=\frac{\ell^{2}}{32 \pi G} \int_{\partial \Sigma} I_{\xi} \omega_{a b}\left(\epsilon_{c d}^{a b} F_{j k}^{c d}-2 \gamma F_{j k}^{a b}\right) d x^{j} \wedge d x^{k}
$$

This generalizes the result of [21], 22], 23], 24], and [25] to the case of first order gravity with Immirzi parameter.

Having the general expression for the charge, we can now turn to finding the formula for the entropy. According to [21] and [22] the black hole entropy $S$ is proportional to the value of the Noether charge (3.5) calculated at the black hole horizon and associated with a timelike Killing vector $\partial / \partial t$, which vanishes at the horizon $\partial \Sigma_{H}$

$$
\left.Q\left(\frac{\partial}{\partial t}\right)\right|_{\partial \Sigma_{H}}=\frac{\kappa}{2 \pi} \text { Entropy }
$$

where $\kappa$ is the surface gravity. The question we would like to address here is how the presence of the Immirzi parameter influence the resulting expression for entropy. In this paper 
we will investigate only the case of AdS-Schwarzschild black hole, leaving another examples of the asymptotically anti-de Sitter black hole spacetimes to the forthcoming publication.

To calculate the value of the Noether charges (3.5) for the Schwarzschild-AdS spacetime let us first fix the metric to be

$$
d s^{2}=-f(r)^{2} d t^{2}+f(r)^{-2} d r^{2}+r^{2}\left(d \theta^{2}+\sin ^{2} \theta d \varphi^{2}\right)
$$

with

$$
f(r)^{2}=\left(1-\frac{2 G M}{r}+\frac{r^{2}}{\ell^{2}}\right) .
$$

It can be checked that for the case of the metric (3.7) the surface gravity $\kappa$ defined by the equation

$$
I_{\xi} \omega^{a b} \xi_{b}=\kappa \xi^{a}
$$

is given by

$$
\kappa=\left.\omega_{t}^{01}\right|_{r_{H}}=\left.\left(\frac{1}{2} \frac{\partial f(r)^{2}}{\partial r}\right)\right|_{r_{H}} \quad T=\frac{\kappa}{2 \pi} .
$$

The charge associated with the timelike Killing vector $\xi \equiv$ $\partial / \partial t$ equals

$$
\begin{aligned}
& Q[\xi]=\frac{4 \ell^{2}}{32 \pi G} \int_{\partial \Sigma} \omega_{t}^{01}\left(\epsilon_{0123} F_{j k}^{23}-\gamma F_{j k 01}\right) d x^{j} \wedge d x^{k}= \\
& \frac{4 \ell^{2}}{32 \pi G} \int_{\partial \Sigma}\left(\frac{1}{2} \frac{\partial f(r)^{2}}{\partial r}\right)\left(1+\frac{r^{2}}{\ell^{2}}-f(r)^{2}\right) \sin \theta d \theta \wedge d \varphi .
\end{aligned}
$$

Notice that this expression does not depend of the Immirzi parameter; the $\gamma$-dependent terms in (3.5) have just dropped out.

The value of this charge calculated at the boundary at infinity gives

$$
Q[\xi]_{\infty}=\lim _{r \rightarrow \infty} \frac{1}{4 \pi} \int_{\partial \Sigma}\left(M+\frac{\ell^{2} G M^{2}}{r^{3}}\right) \sin \theta d \theta \wedge d \varphi=M
$$

as it should be [23], 24].

The charge calculated at the Schwarzschild-AdS black hole horizon equals

$$
\begin{aligned}
Q[\xi]_{H} & =\frac{\kappa \ell^{2}}{8 \pi G}\left(1+\frac{r_{H}^{2}}{\ell^{2}}\right) \int_{\partial \Sigma} \sin \theta d \theta \wedge d \varphi \\
& =\frac{\kappa}{2 \pi} \frac{4 \pi\left(r_{H}^{2}+\ell^{2}\right)}{4 G},
\end{aligned}
$$

where $\kappa$ is the surface gravity defined by eq. 3.10). The horizon radius $r_{H}$ is the largest real solution of the third order equation

$$
r^{3} / \ell^{2}+r-2 G M=0,
$$

which allows us to rewrite the expression (3.13) as

$$
Q[\xi]_{H}=\kappa \frac{M \ell^{2}}{r_{H}} .
$$

From 3.13 it's straightforward to see that the black hole entropy yields the form

$$
\mathrm{S}=\frac{A}{4 G}+\frac{4 \pi \ell^{2}}{4 G}
$$

The first term is the standard Bekenstein-Hawking area law, while the second is just a constant, which does not alter the first law of thermodynamics. In the first order formalism its presence can be regarded as price that has to be paid for the regularization at infinity and the presence of the Euler term in the action. The appearance of the additive constant in the expression for black hole has been discussed in the context of Lovelock and Gauss-Bonet gravity theories e.g. in [26], 27]. It is worth mentioning that our model avoids the problem of the negative entropy 28 .

In the context of the $\mathrm{BF}$ construction presented above this constant could be understood as an indication that the vacuum of the constrained BF theory (being the maximally symmetric spacetime with $S O(2,3)$ symmetry) carries some entropy. A deeper origin of this entropy remains still to be understood.

\section{DISCUSSION: WHAT ABOUT THE IMMIRZI PARAMETER?}

In the precedent section we calculated the entropy of the Schwarzschild-AdS black hole by making use of the WaldIyer prescription. Interestingly, the resulting expression (3.15) does not contain any trace of the Immirzi parameter, in spite of the fact this parameter was present in the Lagrangian of the dynamical theory that we started with.

Before turning to the discussion of this intriguing result let us try to trace the reason for the Immirzi parameter disappearance. Let us consider, for simplicity, the action without the regularizing Euler and Pontryagin terms, using just the Palatini and Holst actions. In the case of axisymmetric stationary spacetime the charge associated with the Killing vector $\partial_{\chi}$ being either $\partial_{t}$ or $\partial_{\varphi}$ (related to the mass and angular momentum at infinity) reads

$$
\begin{aligned}
Q\left[\partial_{\chi}\right] & =\frac{1}{32 \pi G} \int_{\partial \Sigma} \omega_{\chi}^{a b}\left(\epsilon_{a b c d}\left(e_{\theta}^{c} e_{\varphi}^{d}-e_{\varphi}^{c} e_{\theta}^{d}\right)\right. \\
& \left.-\frac{2 \gamma}{32 \pi G} \int_{\partial \Sigma} \omega_{\chi}^{a b}\left(e_{\theta a} e_{\varphi b}-e_{\varphi a} e_{\theta b}\right)\right) .
\end{aligned}
$$

Using the definition of the connection $\omega_{\mu}^{a b}=e^{\nu a} \nabla_{\mu} e_{\nu}^{b}=$ $e^{\nu a}\left(\partial_{\mu} e_{\nu}^{b}-\Gamma_{\mu \nu}^{\lambda} e_{\lambda}^{b}\right)$ we can drastically simplify this formula and for $\chi=t$ we have

$$
Q\left[\partial_{t}\right]=\frac{1}{16 \pi G} \int_{\partial \Sigma}\left(\epsilon_{\theta \varphi}^{\mu \nu} \Gamma_{\mu t \nu}-\gamma\left(\Gamma_{\theta t \varphi}-\Gamma_{\varphi t \theta}\right)\right) .
$$

Therefore Immirzi parameter might be present in the expression for black black hole thermodynamics if

$$
\gamma \int_{\partial \Sigma} \partial_{\theta} g_{t \varphi} \neq 0
$$

Thus we expect that such a contribution proportional to Immirzi parameter can be present in Taub-NUT-AdS spacetime, and it is going to be proportional to Taub-NUT 'mass', and not to the Schwarzschild one. We will present the detailed discussion of several black hole asymptotically AdS spacetimes in the forthcoming paper.

Let us now return to the problem if and how our expression for the entropy 3.15) can be reconciled with the Loop Quantum Gravity calculation [15], [16], 17], 20] according to which the black hole entropy computed by counting the black hole horizon microstates equals

$$
\mathrm{S}_{L Q G}=\frac{\gamma_{M}}{\gamma} \frac{A}{4 G}
$$


where $\gamma_{M}$ is a parameter, whose numerical value is between 0.2 and 0.3 , accompanied by higher order corrections; see 20] for detailed discussion. It is not hard to understand why $\gamma$ should be explicitly present in this formula. Indeed the Immirzi parameter defines the size of the quantum of the area, and therefore it must show up in the state counting for black hole horizon. It would have been for some quite unnatural cancelations to make it disappear from the entropy formula in the semiclassical limit of Loop Quantum Gravity. Yet the expression for entropy presented above (3.15), which holds in the semiclassical theory, whose quantum counterpart LQG is supposed to be, shows no trace of $\gamma$.

A possible way to resolve this dilemma, as suggested in [29], is to notice that the entropy in (4.4) was calculated using microscopic quantities, while in eq. 3.15 with the help of those of effective low energy ones. It follows that there might be highly nontrivial relations between the area $A$ and and Newton's constant $G$ of (4.4) and those of (3.15), so that, when the relations between them are properly understood, and the renormalization effects are taken into account, the two expression may turn out to be completely equivalent.

Another possible way out was proposed recently in [30]. In this paper it was observed that there exist an additional ambiguity parameter associated with the construction of the $S U(2)$ Chern-Simons theory that describes the microscopic degrees of freedom of the isolated horizon. This parameter is of the similar nature as the Immirzi one, and one can adjust the two in such a way, so as to make the final expression for the black hole entropy having the standard Bekenstein-Hawking form.

In both cases it remains to be understood in details how the proposed mechanisms work. This question is related to the notorious problem of the semiclassical limit of Loop Quantum Gravity, and it seems that without controlling this limit one cannot make any definite conclusions.

\section{ACKNOWLEDGEMENTS}

We thank A. Perez for discussion and bringing the paper [30] to our attention. The work of J. Kowalski-Glikman was supported in part by grants $182 / \mathrm{N}-\mathrm{QGG} / 2008 / 0$, and the work of R. Durka was supported by the National Science Centre grant N202 112740.
[1] S. W. MacDowell and F. Mansouri, "Unified Geometric Theory Of Gravity And Supergravity," Phys. Rev. Lett. 38, 739 (1977) [Erratum-ibid. 38, 1376 (1977)].

[2] K. S. Stelle, P. C. West, "Spontaneously Broken De Sitter Symmetry And The Gravitational Holonomy Group," Phys. Rev. D21 (1980) 1466.

[3] J. F. Plebanski, "On the separation of Einsteinian substructures," J. Math. Phys. 18 (1977) 2511-2520.

[4] R. Capovilla, T. Jacobson, J. Dell et al., "Selfdual two forms and gravity," Class. Quant. Grav. 8 (1991) 41-57.

[5] R. Capovilla, T. Jacobson, J. Dell, "A Pure spin connection formulation of gravity," Class. Quant. Grav. 8 (1991) 59-73.

[6] A. Starodubtsev, "Topological excitations around the vacuum of quantum gravity. I: The symmetries of the vacuum," arXiv:hep-th/0306135.

[7] L. Smolin and A. Starodubtsev, "General relativity with a topological phase: An action principle," arXiv:hepth/0311163.

[8] L. Freidel and A. Starodubtsev, "Quantum gravity in terms of topological observables," arXiv:hep-th/0501191.

[9] D. K. Wise, "MacDowell-Mansouri gravity and Cartan geometry," arXiv:gr-qc/0611154.

[10] G. Immirzi, "Real and complex connections for canonical gravity," Class. Quant. Grav. 14 (1997) L177-L181. [grqc/9612030].

[11] R. Durka, J. Kowalski-Glikman, "Hamiltonian analysis of SO $(4,1)$ constrained BF theory," Class. Quant. Grav. 27, 185008 (2010). [arXiv:1003.2412 [gr-qc]].

[12] S. Holst, "Barbero's Hamiltonian derived from a generalized Hilbert-Palatini action," Phys. Rev. D 53 (1996) 5966 [arXiv:gr-qc/9511026].

[13] J. F. Barbero G., "Real Ashtekar variables for Lorentzian signature space times," Phys. Rev. D51 (1995) 55075510. [gr-qc/9410014].
[14] C. Rovelli and L. Smolin, "Discreteness of area and volume in quantum gravity," Nucl. Phys. B 442 (1995) 593 [Erratum-ibid. B 456 (1995) 753] [arXiv:gr-qc/9411005].

[15] A. Ashtekar, J. C. Baez and K. Krasnov, "Quantum geometry of isolated horizons and black hole entropy," Adv. Theor. Math. Phys. 4 (2000) 1 [arXiv:gr-qc/0005126].

[16] M. Domagala and J. Lewandowski, "Black hole entropy from quantum geometry," Class. Quant. Grav. 21 (2004) 5233 [arXiv:gr-qc/0407051].

[17] K. A. Meissner, "Black hole entropy in loop quantum gravity," Class. Quant. Grav. 21 (2004) 5245 [arXiv:grqc/0407052].

[18] J. Engle, A. Perez and K. Noui, "Black hole entropy and SU(2) Chern-Simons theory," Phys. Rev. Lett. 105 (2010) 031302 [arXiv:0905.3168 [gr-qc]].

[19] J. Engle, K. Noui, A. Perez and D. Pranzetti, "Black hole entropy from an SU(2)-invariant formulation of Type I isolated horizons," Phys. Rev. D 82, 044050 (2010) [arXiv:1006.0634 [gr-qc]].

[20] I. Agullo, J. Fernando Barbero, E. F. Borja, J. Diaz-Polo, E. J. S. Villasenor, "Detailed black hole state counting in loop quantum gravity," Phys. Rev. D82 (2010) 084029. [arXiv:1101.3660 [gr-qc]].

[21] R. M. Wald, "Black hole entropy is the Noether charge," Phys. Rev. D 48, 3427 (1993) [arXiv:gr-qc/9307038].

[22] V. Iyer and R. M. Wald, "A Comparison of Noether charge and Euclidean methods for computing the entropy of stationary black holes," Phys. Rev. D 52, 4430 (1995) [arXiv:gr-qc/9503052].

[23] R. Aros, M. Contreras, R. Olea, R. Troncoso and J. Zanelli, "Conserved charges for even dimensional asymptotically AdS gravity Phys. Rev. D 62, 044002 (2000) [arXiv:hep-th/9912045].

[24] R. Aros, M. Contreras, R. Olea, R. Troncoso and J. Zanelli, "Conserved charges for gravity with locally AdS asymptotics," Phys. Rev. Lett. 84, 1647 (2000) 
[arXiv:gr-qc/9909015].

[25] R. Olea, "Mass, angular momentum and thermodynamics in four-dimensional Kerr-AdS black holes," JHEP 0506 (2005) 023 [arXiv:hep-th/0504233].

[26] C. Krishnan, S. Kuperstein, "A Comment on Kerr-CFT and Wald Entropy," Phys. Lett. B677, 326-331 (2009). [arXiv:0903.2169 [hep-th]].

[27] S. Sarkar, A. C. Wall, "Second Law Violations in Lovelock Gravity," [arXiv:1011.4988 [gr-qc]].

[28] T. Clunan, S. F. Ross, D. J. Smith, "On Gauss-Bonnet black hole entropy," Class. Quant. Grav. 21, 3447-3458
(2004). [gr-qc/0402044].

[29] T. Jacobson, "Renormalization and black hole entropy in Loop Quantum Gravity," Class. Quant. Grav. 24 (2007) 4875-4879. [arXiv:0707.4026 [gr-qc]].

[30] A. Perez, D. Pranzetti, "Static isolated horizons: SU(2) invariant phase space, quantization, and black hole entropy," Entropy, 13, (2011) 744 [arXiv:1011.2961 [gr-qc]]. 